\title{
Endometriosis Diagnosis Correlation of Laparoscopic Visualization and Histopathology Confirmation in Low Resource Setting
}

\author{
J. Wanyoike Gichuhi ${ }^{*}$, Julius Ogengo ${ }^{2}$, Peter Gichangi \\ ${ }^{1}$ Department of Obstetrics \& Gynaecology, University of Nairobi, Nairobi, Kenya \\ ${ }^{2}$ Department of Anatomy, University of Nairobi, Nairobi, Kenya \\ Email: ^drjoewanyoike@yahoo.co.uk
}

How to cite this paper: Gichuhi, J.W., Ogengo, J. and Gichangi, P. (2021) Endometriosis Diagnosis Correlation of Laparoscopic Visualization and Histopathology Confirmation in Low Resource Setting. Open Journal of Obstetrics and Gynecology, 11, 845-852.

https://doi.org/10.4236/ojog.2021.117079

Received: June 8, 2021

Accepted: July 13, 2021

Published: July 16, 2021

Copyright $\odot 2021$ by author(s) and Scientific Research Publishing Inc. This work is licensed under the Creative Commons Attribution International License (CC BY 4.0).

http://creativecommons.org/licenses/by/4.0/

\begin{abstract}
Background: Endometriosis is enigmatic clinical entity which is described as the location of the endometrial tissue external of the uterine cavity. Endometriosis constitutes a serious health issue due to its high affliction of $10 \%$ of reproductive women. With limited resources in Africa, it is essential to assess whether diagnosis of endometriosis by laparoscopic visualization can be used as a substitute for histology. Objective: To correlate the diagnosis of endometriosis by laparoscopic visualization and the histological confirmation of the biopsy taken. Methods: A prospective cross sectional study with a sample size of 443 was undertaken in the diagnosis of endometriosis among Africans in Nairobi, Kenya from March 2019 to March 2021. Women undergoing laparoscopy were screened for endometriosis by visualization and a biopsy was taken for histopathology. Diagnosis of endometriosis by visualization was correlated with histological confirmed endometriosis. Results: Women with a diagnosis of endometriosis through laparoscopic visualization were found to be $77(17.4 \%)$ and 30 (6.8\%) had positive histology for endometriosis. Laparoscopic visualization diagnosis had a low positive predictive value of $39 \%$. Conclusion: Laparoscopic visualization diagnosis had a low positive predictive value of $39 \%$ and this did not correlate with histopathologic diagnosis. It is essential to perform biopsy with histopathology for the confirmation of endometriosis.
\end{abstract}

\section{Keywords}

Endometriosis, Diagnosis, Laparoscopy, Africa 


\section{Background}

Endometriosis is a woman's reproductive ailment, described as presence of endometriotic glands and stroma outside the endometrial cavity, mainly in the pelvic peritoneum, ovary and rectovaginal septum. Endometriosis is a crippling ailment that affects the quality of life and afflicts $6 \%-10 \%$ of women and its symptomatology encompasses mainly chronic pelvic pain, dysmenorrhoea, dyspareunia and infertility and hence it is one of the most frequent gynaecological ailments [1]. Endometriosis manifests with chronic pelvic pain and infertility, and both have high prevalence of as high as $35 \%-50 \%$ [2]. In first world countries, there is clear documentation of the diagnosed endometriosis, however, in the developing countries, there are scanty documentations [3]. Endometriosis has been exhaustively investigated in the developed world, however, this is not the case in Africa. The understanding, diagnosis and establishment of the disease burden of endometriosis in Africa are essential for the appropriate management of this disease. There is a conception that African women are hardly inflicted with endometriosis and that is most likely due to poor diagnosis methodology [3] [4]. There is scanty literature on Africans on the endometriosis clinical presentation and the prevalence [3]. Diagnosis of endometriosis can only be effectively performed through laparoscopy or laparotomy; limitation of laparoscopic facilities that are essential in diagnosis and treatment in Africa and lack of awareness of the impact of endometriosis may account for low detection and hence prevalence of endometriosis in African [3] [5]. The gold standard for the diagnosis of endometriosis is laparoscopic visualization and histological confirmation and this has revolutionized the management of this disease [6] [7]. Laparoscopic visualization diagnosis of endometriosis has been documented to have positively predictive value of $45 \%$ [8]. Endometriosis can mimic other pathologies such as pelvic infection, endosalpingiosis and cancer in clinical appearance and therefore better laparoscopic optics resolution, recognition of various clinical manifestations of endometriosis and methodological assessment of the pelvic anatomy are imperative [9].

\section{Objective}

To determine the correlation of endometriosis diagnosis by laparoscopic visualization and histopathology confirmation in indigenous African women.

\section{Methods}

This was an analytical cross-selection study with a sample size of 443 patients. The sample size was determined by using the statistical formula of Fisher method. According to studies undertaken in similar settings, the prevalence of endometriosis has been reported to range from $5 \%-10 \%$. A conservative estimate of $10 \%$ that gives the largest possible sample size will be used for purposes of sample size calculation. The study site was undertaken in 2 purposively selected hospitals across Nairobi city to ensure ownership and varying levels of specialty, 
that is Kenyatta National Hospital And the Nairobi hospital. The study population was women aged 18 - 49 years undergoing laparoscopic surgery in any of the two participating hospitals and willing to be part of the study. Data was collected using a structured questionnaire and included all case records for patients undergoing laparoscopic surgery. The structured questionnaire was designed by the authors and pretested for validity and reliability. The respondents were informed about the study, its objectives, risks and benefits. The willing participants were requested to provide written consent. The structured questionnaire was completed by the clinician preparing the patient for surgery while completeness and follow-up for any missing information were done by the principle investigator. The patients were reviewed pre-operatively for the history, clinical presentations and investigations. The anatomical location and staging of the endometriosis were documented. The extent of the endometriosis was described using revised America Society for reproductive Medicine (Revised ASM) [7]. Subtle lesion that might represent endometriosis were excised even if endometriosis was not suspected. One to four biopsies were taken from each patient. Histological confirmation of endometriosis was done by staining samples with haematoxylin and eosin. All data obtained from the questionnaire was verified and had double entered into a computer using Microsoft Access database. The data was analyzed using Social SPSS version 22.0. The chi-square and logistic regression were used to determine the predictors of endometriosis among women undergoing laparoscopic surgery (Tables 1-5 and Figure 1).

Table 1. Laparoscopic procedure $\mathrm{n}=443$.

\begin{tabular}{ccc}
\hline Surgical Procedure & \multicolumn{2}{c}{ Total } \\
\cline { 2 - 3 } Excision of Endometriotic Tissue & $\mathbf{n}$ & $\%$ \\
Diagnostic Laparoscopy & 30 & 6.8 \\
Adhesionlysis & 71 & 33.2 \\
Ovarian Cystectomy & 67 & 15.1 \\
Oophorectomy & 54 & 12.2 \\
Myomectomy & 5 & 1.1 \\
Tuboplasty & 52 & 11.7 \\
Salpingectomy & 57 & 12.9 \\
Retrieval of IUD & 20 & 4.5 \\
Drainage of Abscess & 9 & 2.0 \\
Hysterectomy & 4 & .9 \\
Appendectomy & 52 & 11.7 \\
Ovarian Drill & 5 & 1.1 \\
\hline
\end{tabular}

The main laparoscopic surgeries performed were diagnostic (16\%), Adhesionalysis (15.1\%), Tuboplasty (18.7\%), ovarian cystectomy (12.2\%), Myomectomy (11.7\%), Hysterectomy (11.7\%), excision of endometriosis was $6.8 \%$, Salpingectomy (4.5\%), Retrieval of lost IUD (2.0\%), appendectomy (1.1\%), oophorectomy (1.1\%), and ovarian drill (0.6\%). 
Table 2. Histological findings on laparoscopic biopsies $(n=443)$.

\begin{tabular}{ccc}
\hline & $\mathbf{n}$ & $\%$ \\
\hline Adenomyosis & 8 & 1.8 \\
Appendicitis & 5 & 1.1 \\
Cervical dysplasia & 1 & 0.2 \\
Clinical endometriosis but histologically no endometriosis & 24 & 5.4 \\
Confirmed histological endometriosis & 30 & 6.8 \\
Ectopic pregnancy & 4 & 0.9 \\
Endometrial hyperplasia & 1 & 0.2 \\
Fallopian tubes & 23 & 5.2 \\
Myoma & 88 & 19.9 \\
No pathology & 196 & 44.2 \\
Ovarian cyst & 45 & 10.2 \\
Ovarian malignancy & 4 & 0.9 \\
Teratoma & 5 & 1.1 \\
\hline
\end{tabular}

The prevalence of histological confirmed endometriosis was $6.8 \%$, however the most common pathology was myoma (19.9\%) and $44.2 \%$ of women had no pathology detected histologically.

Table 3. Signs of Endometriosis $(\mathrm{n}=30)$.

\begin{tabular}{ccc}
\hline Signs of Endometriosis & n & $\%$ \\
\hline Puckered blue-black & 6 & 20.0 \\
Powder-burned appearance & 10 & 33.3 \\
Subtle (Popular, Glandular, vesicular) & 3 & 10.0 \\
Haemorrhagic (Red vesicular or Flame-like) & 8 & 26.7 \\
Fibrotic lesions (White to black pigmented). & 9 & 30.0 \\
Chocolate cyst/endometrioma. & 8 & 26.7 \\
Deep Infiltrating Endometriosis & 8 & 26.7 \\
Extra pelvic & 2 & 6.7 \\
\hline
\end{tabular}

The most common form of presentation in superficial endometriosis was powder-burned appearance (33\%) followed by fibrotic lesions (White to black pigmented), (30\%), puckered blue-black (20\%), subtle (10\%) whilst endometrioma and deep infiltrating endometriosis accounted for $26.7 \%$ with extra pelvic endometriosis being $2 \%$.

Table 4. Anatomical site of Endometriosis $(\mathrm{n}=30)$.

\begin{tabular}{ccc}
\hline & $\mathbf{n}$ & $\%$ \\
\hline Anatomic site of endometriosis & & \\
Anterior uterine & 2 & 6.7 \\
Extra pelvic site & 1 & 3.3 \\
Gut & 1 & 3.3 \\
\hline
\end{tabular}




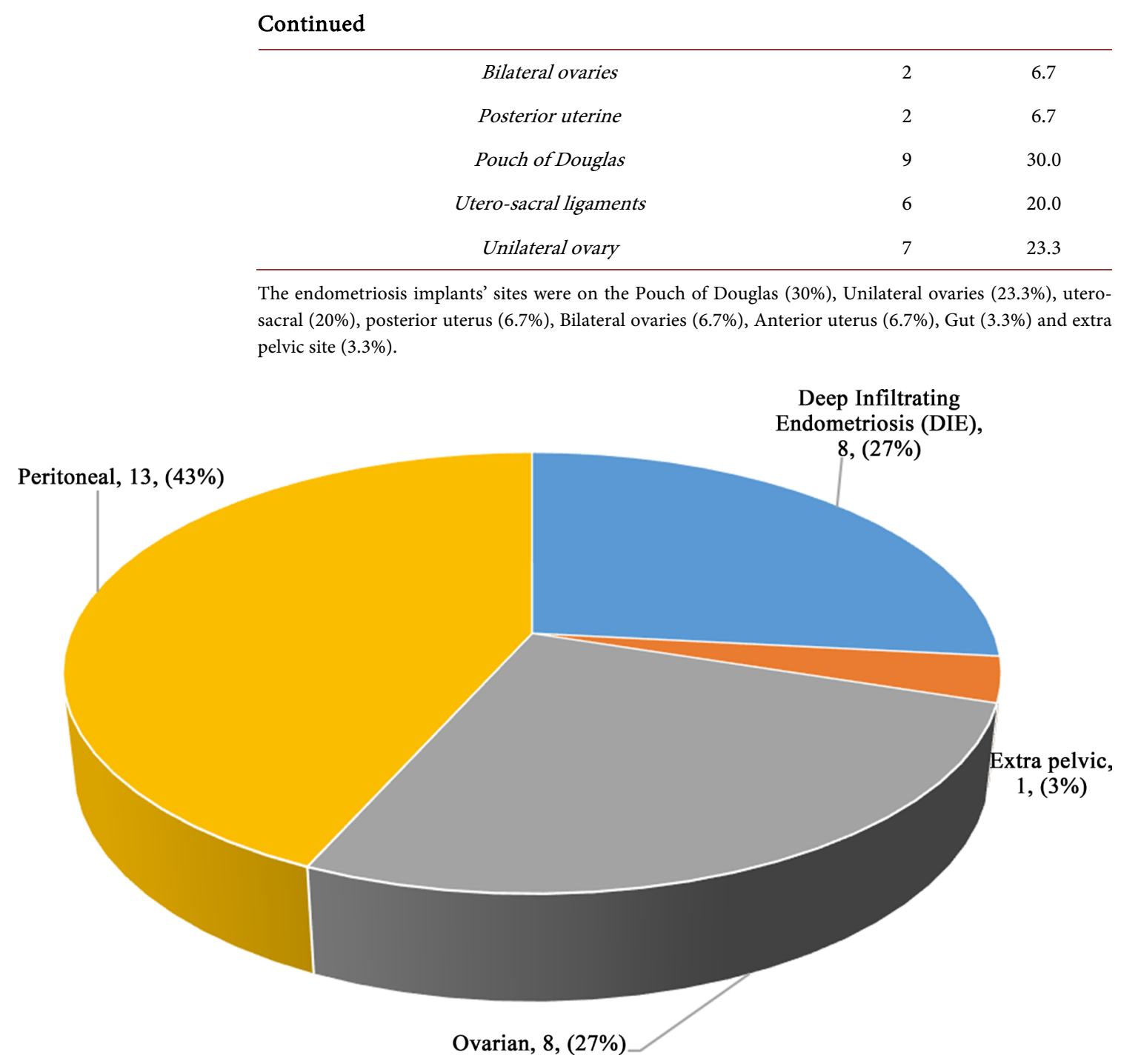

The majority of the histological confirmed endometriosis were superficial endometriosis (43\%), with ovarian and deep infiltrating endometrioma each having $27 \%$ and extra pelvic endometriosis havening $3 \%$.

Figure 1. Classification of Endometriosis Status $(\mathrm{n}=30)$.

Table 5. Histological confirmed endometriosis.

\begin{tabular}{ccc}
\hline & NO & $\%$ \\
\hline Confirmed histological endometriosis & 30 & $6.8 \%$ \\
Clinical endometriosis by visualization & 77 & $17.4 \%$ \\
\hline
\end{tabular}

Endometriosis clinical diagnosed by visualization was 77 (17.4\%) and out of this $30(6.8 \%)$ were histologically confirmed.

\section{Discussion}

The mean age of the women in the study was 33 years and there was no statistical difference between the women with endometriosis and those without.

Endometriosis clinically diagnosed by visualization was 77 (17.4\%) and out of this only $30(6.8 \%)$ were histologically confirmed as endometriosis. Laparoscopic 
visualization diagnosis had a low positive predictive value of 39\%. This was lower than other studies study that found laparoscopic visualization diagnosis of endometriosis to have positive predictive value of $45 \%$ and $63.8 \%$ [8] [10].

The prevalence of endometriosis has been speculated to be as high as $10 \%$ in the women of reproductive age, however, the findings in this study showed a lower prevalence [5]. This study was not consisted with a Nigerian study in Ibadan where the prevalence of endometriosis with only laparoscopic visualization and no histological examination was found to be $48.8 \%$ [11]. This study showed the prevalence of endometriosis with laparoscopic visualization to be 17.4\%. This study was not consisted with Chapmans laparoscopic studies in African American women, where he found prevalence of histologically confirmed endometriosis to be $21 \%$ in women who had had pelvic inflammatory disease [12].

The main laparoscopic surgery performed were diagnostic (33.2\%), Adhesionalysis $(15.1 \%)$, Tuboplasty (18.7\%), Ovarian cystectomy (12.2\%), Myomectomy (11.7\%), Hysterectomy (11.7\%), excision of endometriosis (6.8\%, Salpingectomy (4.5\%), Retrieval of lost IUD (2.0\%), appendectomy (1.1\%), oophorectomy (1.1\%), and ovarian drill (0.6\%).

The sites of the endometriosis implants were on the Pouch of Douglas (30\%), Unilateral ovaries (23.3\%), uterosacral (20\%), posterior uterus (6.7\%), Bilateral ovaries (6.7\%), Anterior uterus (6.7\%), Gut (3.3\%) and extra pelvic site $(3.3 \%)$. This was consisted with a study by Wanyonyi et al. [10] who found most endometriotic positive biopsies biopsies were found in the ovary and posterior cul-de-sac [10].

The majority of the histological confirmed endometriosis were superficial endometriosis (43\%), with ovarian and deep infiltrating endometrioma each having $(27 \%)$ and extra pelvic endometriosis having (2\%). The most common form of presentation of superficial endometriosis was powder-burned appearance (33\%) followed by fibrotic lesions (30\%), puckered blue-black (20\%) and subtle $(10 \%)$.

The overall histopathology findings were confirmed histological endometriosis $(6.8 \%)$, myoma (19.9\%), ovarian cyst (10.2\%), fallopian tubes (5.2\%)., adenomyosis $(1.8 \%)$, teratoma $(1.1 \%$,$) appendicitis (1.1 \%)$, ectopic pregnancy $(0.9 \%)$, ovarian malignancy $(0.9 \%)$, endometrial hyperplasia $(0.2 \%)$, cervical dysplasia (0.2\%) and no pathology (44.2\%). Laparoscopic visualization of endometriosis among Africans in low resource setting does not correlate with histopathologic diagnosis due to presence of multiple pathologies which may be diagnosed as endometriosis by clinical appearance. It is essential to perform biopsy with histopathology for confirmation of endometriosis.

\section{Conclusion}

The endometriosis diagnosed by laparoscopic visualization was $17.4 \%$ and the histological confirmed endometriosis was $6.8 \%$. Laparoscopic visualization di- 
agnosis had a low positive predictive value of $39 \%$ and did not correlate with histopathologic diagnosis due to presence of multiple lesions which may mimic endometriosis on laparoscopic visualization. For definitive diagnosis of endometriosis performance of biopsy with histopathological confirmation is imperative.

\section{Conflicts of Interest}

The authors declare no conflicts of interest regarding the publication of this paper.

\section{References}

[1] Burney, R.O. and Giudice, L.C. (2012) Pathogenesis and Pathophysiology of Endometriosis. Fertility and Sterility, 98, 511-519.

https://doi.org/10.1016/j.fertnstert.2012.06.029

[2] Meuleman, C., Vandenabeele, B., Fieuws, S., et al. (2009) High Prevalence of Endometriosis in Infertile Women with Normal Ovulation and Normaspermic Partners. Fertility and Sterility, 92, 68-74. https://doi.org/10.1016/j.fertnstert.2008.04.056

[3] Kyema, C.M., Mwenda, J.W., Machoki, J., et al. (2007) Endometriosis in African Women. Women's Health, 3, 629-635. https://doi.org/10.2217/174555057.3.5.629

[4] Thacher, T.D., Nwana, E.J.C. and Karshima, J.A. (1997) Extrapelvic Endometriosis in Nigeria. International Journal of Gynecology \& Obstetrics, 57, 57-58. https://doi.org/10.1016/S0020-7292(97)00116-1

[5] Eskenazi, B. and Warner, M.L. (1997) Epidemiology of Endometriosis. Obstetrics and Gynecology Clinics of North America, 24, 2. https://doi.org/10.1016/S0889-8545(05)70302-8

[6] Sasson, I.E. and Taylor, H.S. (2008) Stem Cells and the Pathogenesis of Endometriosis. Annals of the New York Academy of Sciences, 1127, 106-115. https://doi.org/10.1196/annals.1434.014

[7] American Society for Reproductive Medicine (ASRM) (2006) Revised American Society for Reproductive Medicine Classification of Endometriosis. Fertility and Sterility, 67, 817-821. https://doi.org/10.1016/S0015-0282(97)81391-X

[8] Walter, A.J., Herntz, J.G., Magtiba, P.M., Cornell, J.L. and Magrina, J.F. (2001) Endometriosis: Correlation between Histologic and Visual Findings at Laparoscopy. American Journal of Obstetrics \& Gynecology, 184, 1407-1413. https://doi.org/10.1067/mob.2001.115747

[9] Martin, D.C., Hubert, G.D., Vander, Z., et al. (1989) Laparoscopic Appearances of Peritoneal Endometriosis. Fertility and Sterility, 51, 63-67. https://doi.org/10.1016/S0015-0282(16)60429-6

[10] Wanyonyi, S.Z., Sequeira, E. and Mukono, S.G. (2011) Correlation between Laparoscopic and Histological Diagnosis of Endometriosis. International Journal of Gynecology \& Obstetrics, 115, 273-276. https://doi.org/10.1016/j.ijgo.2011.07.022

[11] Fawole, A.O., Bello, F.A., Ogubonde, O., OduKogbe, A.O., Nkwocha, G.C., Nnoham, K.E., Zondervan, K.T., Akintan, A., Abdus-Salam, R.A. and Okunlola, M.A. (2015) Endometriosis and Associated Symptoms among Nigerian Women. International Journal of Gynecology \& Obstetrics, 130, 190-194. 
https://doi.org/10.1016/j.ijgo.2015.02.030

[12] Chatman, D. (1976) Endometriosis in the Black Woman. American Journal of Obstetrics and Gynecology, 125, 987-989.

https://doi.org/10.1016/0002-9378(76)90502-0 